\title{
Crossed Product Decompositions of a Purely Infinite Von Neumann Algebra with Faithful, Almost Periodic Weight
}

\author{
KenNeth DyKema
}

\begin{abstract}
For $\mathcal{M}$ a separable, purely infinite von Neumann algebra with almost periodic weight $\varphi$, Connes' decomposition of $\mathcal{M}$ as a crossed product of a semifinite von Neumann algebra by a trace-scaling action of a countable abelian group is described, and factoriality is related to ergodicity of a certain partial action. Then Takasaki's continuous decomposition of the same algebra is related to the above discrete decomposition via Takesaki's notion of induced action, but here one induces up from a dense subgroup. The above results are used to give a model for the one-parameter trace-scaling action of $\mathbf{R}_{+}^{*}$ on the injective $\mathrm{II}_{\infty}$ factor. Finally, another model of the same action, due to work of Aubert and explained by Jones, is described.
\end{abstract}

Introduction. A crucial part of the present-day understanding of type III factors is their decomposition as crossed products of type $\mathrm{II}_{\infty}$ von Neumann algebras by groups of trace-scaling (or trace-decreasing) automorphisms. This was accomplished by Connes in [4] and by Takesaki in [15]. Connes defined the classification of type III factors as type $\mathrm{III}_{\lambda}, 0 \leq \lambda \leq 1$ and showed that a type $\mathrm{III}_{\lambda}$ factor where $0 \leq \lambda<1$ is isomorphic to the crossed product of a type $\mathrm{II}_{\infty}$ von Neumann algebra, $\mathcal{N}$, by a single automorphism (i.e., by the group $\mathbf{Z})$. When $\lambda>0, \mathcal{N}$ can be chosen to be a factor and the automorphism tracescaling, and in the case $\lambda=0$ the automorphism is ergodic on the center of $\mathcal{N}$ and a trace can be chosen such that the automorphism is strictly decreasing of the trace.

Takesaki developed the theory of crossed products of a von Neumann algebra by actions of locally compact groups, including his duality theory and his theory of induced actions. He thereby proved the continuous decomposition for a factor $\mathcal{M}$ : if $\mathcal{M}$ is type $\mathrm{III}_{1}$, then $\mathcal{M}$ is the crossed product of a type $\mathrm{II}_{\infty}$ factor $\mathcal{N}$ by a one-parameter group of trace-scaling automorphisms. 
Almost periodic weights (the definition is reviewed in Section 1.3) were defined by Connes in [4] and can be used to elucidate the structure of certain type III $_{1}$ factors. Connes defined the invariant $S d$ for a full type III $_{1}$ factor in terms of its almost periodic weights in $[\mathbf{6}]$, where he also showed that there is a type $\mathrm{III}_{1}$ factor having no almost periodic weights. However, many type $\mathrm{III}_{1}$ factors of interest have them. For example, the injective type $\mathrm{III}_{1}$ factor, which was shown to be unique by Haagerup [10], has many almost periodic weights, (cf Section 4). Also, the free products of certain finite dimensional algebras with respect to certain states that are not traces are known to be type $\mathrm{III}_{1}$ factors (see $[\mathbf{3}],[\mathbf{7}]$ and $[\mathbf{8}]$ ) and the free product states on them are almost periodic.

In this paper, given a purely infinite, separable von Neumann algebra, $\mathcal{M}$, with almost periodic weight, $\varphi$, we recall how results of Connes imply that $\mathcal{M}$ can be written as a discrete crossed product of a type $\mathrm{II}_{\infty}$ von Neumann algebra $\mathcal{N}$ by the action of a countable abelian group.

The main contribution of this paper (Corollary 3.4) is to describe the continuous decomposition for $\mathcal{M}$ in terms of the above discrete decomposition via a straightforward generalization of Takesaki's definition of induced action (Definition 3.1). This generalization is to induce up from a possibly dense subgroup $H \subseteq K$ rather than just a closed subgroup. Of course, one must consider a topology on $H$, different from the restriction of the topology on $K$, with respect to which $H$ is locally compact. One thereby obtains, when $\mathcal{M}$ is a type $\mathrm{III}_{1}$ factor, a description of the continuous core type $\mathrm{II}_{\infty}$ factor and the one-parameter group action on it. This description will be interesting for the study free product factors of type $\mathrm{III}_{1},[8]$.

However, also in the injective case, a concrete description of the (unique by Haagerup [10]) one-parameter trace-scaling action on the (unique by Connes [5]) injective $\mathrm{II}_{\infty}$ factor is obtained. It was explained to the author by V.F.R. Jones that there is another description of this action, which will be described in the last section of this paper.

This paper has five sections. In Section 1, some preliminaries are briefly reviewed and our notation is explained, including Section 1.1, Takesaki's definition of crossed products; Section 1.2, the Arveson-Connes spectral analysis for a compact abelian group action; Section 1.3, Connes' compact group action on a von Neumann algebra with almost periodic weight. In Section 2, the discrete crossed product decomposition for a purely infinite von Neumann algebra with almost periodic weight is described, and factoriality is related to ergodicity of a certain partial action. In Section 3, Takesaki's continuous decomposition for a purely infinite von Neumann algebra with almost periodic weight is shown to arise via an induced action from the discrete action of Section 2. In Section 4, the previous results are used to give a concrete description of the one-parameter trace-scaling action on the injective $\mathrm{II}_{\infty}$ factor. In Section 5 , the previously known description of this action is briefly described. 
Acknowledgements. The work embodied in this paper was prompted by a desire to understand the one-parameter trace-scaling action on the injective $\mathrm{II}_{\infty}$ factor. This desire in turn stemmed from a discussion with M. Takesaki, for which I am grateful. I would also like to thank J. Feldman for helpful discussions and V.F.R. Jones for telling me about the model in Section 5.

1. Preliminaries and notation. Throughout this paper, all groups and Hilbert spaces will be separable and all von Neumann algebras will have separable preduals. Although these restrictions avoid measure theoretic difficulties, it is likely that similar results hold in more general circumstances.

1.1. Crossed products (Takesaki $[\mathbf{1 5}])$. Let $\mathcal{M}$ be a von Neumann algebra and $\alpha: G \rightarrow \operatorname{Aut}(\mathcal{M})$ a continuous action (Arveson [1]) of a locally compact group $G$ on $\mathcal{M}$. (Henceforth, the word "action" will be used to mean a continuous action.) Suppose $\sigma$ is a normal, faithful representation of $\mathcal{M}$ as operators on the Hilbert space $\mathcal{H}$. Consider the von Neumann algebra acting on $L^{2}(G) \otimes \mathcal{H}$ that is generated by

$$
\left\{\lambda_{g} \otimes 1 \mid g \in G\right\} \cup\left\{\pi_{\alpha, \sigma}(a) \mid a \in \mathcal{M}\right\},
$$

where $\lambda_{g} \xi(h)=\xi\left(g^{-1} h\right)$ for $\xi \in L^{2}(G)$ and $g, h \in G$, and where $\pi_{\alpha, \sigma}(a) \zeta(h)=$ $\alpha_{h^{-1}}(a) \zeta(h)$ for $h \in G$ and $\zeta \in L^{2}(G, \mathcal{H})$, where $L^{2}(G, \mathcal{H})$ is the space of squareintegrable, measurable functions from $G$ into $\mathcal{H}$, and is identified with $L^{2}(G) \otimes$ $\mathcal{H}$. The measure on $G$ is left Haar measure. Takesaki proved that the choice of representation $\sigma$ does not affect the isomorphism class, so that one may define the crossed product von Neumann algebra, which we will denote $\mathcal{M} \rtimes_{\alpha} G$, to be generated by elements denoted

$$
\left\{\lambda_{g} \otimes 1 \mid g \in G\right\} \cup\left\{\pi_{\alpha}(a) \mid a \in \mathcal{M}\right\}
$$

in such a way that if $\sigma$ is a representation of $\mathcal{M}$ as above, then there is a faithful, normal representation of $\mathcal{M} \rtimes_{\alpha} G$ given by sending $\lambda_{g} \otimes 1$ to $\lambda_{g} \otimes 1$ and $\pi_{\alpha}(a)$ to $\pi_{\alpha, \sigma}(a)$.

For $G$ abelian, Takesaki defined the dual action $\hat{\alpha}$ of $\hat{G}$ on $\mathcal{M} \rtimes_{\alpha} G$ to be given by $\hat{\alpha}_{\gamma}\left(\lambda_{g} \otimes 1\right)=\overline{\langle g, \gamma\rangle} \lambda_{g} \otimes 1$ and $\hat{\alpha}_{\gamma}\left(\pi_{\alpha}(a)\right)=\pi_{\alpha}(a)$. Takesaki duality is the theorem that

$$
\left(\mathcal{M} \rtimes_{\alpha} G\right) \rtimes_{\hat{\alpha}} \hat{G} \simeq \mathcal{M} \otimes B\left(L^{2}(G)\right) .
$$

If $\mathcal{M}$ is a purely infinite von Neumann algebra with normal, faithful, semifinite weight $\varphi$, let $\sigma^{\varphi}$ be the modular action of $\mathbf{R}$ on $\mathcal{M}$. Let $\mathbf{R}_{+}^{*}$ be the dual group of $\mathbf{R}$ with the pairing $\langle t, \mu\rangle=\mu^{i t}$. Then by Takesaki duality, $\mathcal{M} \simeq$ $\mathcal{M} \otimes B\left(L^{2}(\mathbf{R})\right) \simeq\left(\mathcal{M} \rtimes_{\sigma^{\varphi}} \mathbf{R}\right) \rtimes_{\widehat{\sigma^{\varphi}}} \mathbf{R}_{+}^{*}$. Takesaki showed that $\mathcal{M}_{0}=\mathcal{M} \rtimes_{\sigma^{\varphi}} \mathbf{R}$ is a type $\mathrm{II}_{\infty}$ von Neumann algebra that admits a n.f.s. trace $\tau$ satisfying 
$\tau \circ{\widehat{\sigma^{\varphi}}}_{\mu}=\mu^{-1} \tau$, and that the $\mathrm{W}^{*}$-dynamical system $\left(\mathcal{M}_{0}, \mathbf{R}_{+}^{*}, \widehat{\sigma^{\varphi}}\right)$ does not depend on $\varphi$ (up to isomorphism). We will refer to this dynamical system, giving rise to $\mathcal{M} \simeq \mathcal{M}_{0} \rtimes_{\widehat{\sigma} \varphi} \mathbf{R}_{+}^{*}$, as Takesaki's continuous (crossed product) decomposition of $\mathcal{M}$. If $\mathcal{M}$ is a type III $_{1}$ factor, then $\mathcal{M}_{0}$ is a factor and this is the crossed product decomposition of a type $\mathrm{III}_{1}$ factor that was mentioned in the introduction.

1.2. The Arveson-Connes spectral analysis for a compact abelian group action (Arveson [1] and Connes [4]). Let $G$ be a compact abelian group and let $\Gamma$ denote its dual group, with pairing $\langle g, \gamma\rangle,(g \in G, \gamma \in \Gamma)$. For $\gamma \in \Gamma$ the Arveson-Connes spectral subspace for an action, $\alpha$, of $G$ on $\mathcal{M}$ is denoted $\mathcal{M}^{\alpha}(\gamma)$. Hence $\mathcal{M}^{\alpha}\left(1_{\Gamma}\right)$ is the fixed-point subalgebra of $\mathcal{M}$ under $\alpha$, also simply denoted $\mathcal{M}^{\alpha}$, and moreover

$$
\mathcal{M}^{\alpha}\left(\gamma_{1}\right) \mathcal{M}^{\alpha}\left(\gamma_{2}\right) \subseteq \mathcal{M}^{\alpha}\left(\gamma_{1} \gamma_{2}\right)
$$

and

$$
\mathcal{M}^{\alpha}(\gamma)^{*}=\mathcal{M}^{\alpha}\left(\gamma^{-1}\right)
$$

Also, for $a \in \mathcal{M}$ let

$$
E_{\gamma}(a)=\int_{G}\langle g, \gamma\rangle \alpha_{g}(a) d g,
$$

where $d g$ is Haar measure.

Lemma 1.2.1. For $a \in \mathcal{M}$ the following are equivalent:

(i) $a \in \mathcal{M}^{\alpha}(\gamma)$

(ii) $\alpha_{g}(a)=\overline{\langle g, \gamma\rangle} a \forall g \in G$

(iii) $E_{\gamma}(a)=a$.

Proof. The implication (i) $\Rightarrow$ (ii) is an easy case of Connes [4], Lemma 2.3.5 and (ii) $\Rightarrow$ (i) follows directly from the definition of $\mathcal{M}^{\alpha}(\gamma)$. The equivalence of (ii) and (iii) is easily verified.

Lemma 1.2.2. $E_{\gamma}$ is a weak $k^{*}$-continuous projection of norm 1 from $\mathcal{M}$ onto $\mathcal{M}^{\alpha}(\gamma)$. If $a \in \mathcal{M}, b \in \mathcal{M}^{\alpha}\left(\gamma_{1}\right)$ and $c \in \mathcal{M}^{\alpha}\left(\gamma_{2}\right)$ then

$$
E_{\gamma}(b a c)=b E_{\gamma_{1}^{-1} \gamma \gamma_{2}^{-1}}(a) c .
$$

Proof. In the notation of Connes [4] Section 2.1, $E_{\gamma}=\alpha(\langle\cdot, \gamma\rangle)$, hence is a weak* continuous linear mapping from $\mathcal{M}$ into $\mathcal{M}$, It is clearly of norm 1 . That $E_{\gamma} \circ E_{\gamma}=E_{\gamma}$ is easily verified from the definition. That $E_{\gamma}$ is onto $\mathcal{M}^{\alpha}(\gamma)$ follows from Lemma 1.2.1(iii). Equation (3) holds because $\alpha_{g}($ bac $)=$ $\left\langle g, \gamma_{1} \gamma_{2}\right\rangle b \alpha_{g}(a) c$. 
It makes sense to think of $E_{\gamma}(a)$ as the $\gamma$ th term in the Fourier series for $a$. To see that this generalizes the usual notion of Fourier series, let $\mathcal{M}=L^{\infty}(\mathbf{T})$, $G=\mathbf{T}$ and $\alpha_{z}=$ rotation by $z$.

Lemma 1.2.3. The linear span of $\bigcup_{\gamma \in \Gamma} \mathcal{M}^{\alpha}(\gamma)$ is a strongly dense *subalgebra of $\mathcal{M}$.

Proof. From (1) and (2) it is clear that $\bigcup \mathcal{M}^{\alpha}(\gamma)$ is a ${ }^{*}$-subalgebra of $\mathcal{M}$. Suppose $a \in \mathcal{M}$ is such that $\operatorname{Sp}_{\alpha}(a)$ is a finite subset of $\Gamma$. Consider the function on $G, f(g)=\sum_{\gamma \in \operatorname{Sp}_{\alpha}(a)}\langle g, \gamma\rangle$. Then $\alpha(f) a=\sum_{\gamma \in \operatorname{Sp}_{\alpha}(a)} E_{\gamma}(a)$, and since the Fourier transform $\hat{f}$ is identically 1 on $\operatorname{Sp}_{\alpha}(a)$ and zero elsewhere, it follows from Connes [4] Lemma 2.1.3(b) that $a=\sum E_{\gamma}(a) \in \operatorname{span} \bigcup_{\gamma \in \Gamma} \mathcal{M}^{\alpha}(\gamma)$. But the collection of $a \in \mathcal{M}$ such that $\operatorname{Sp}_{\alpha}(a)$ is finite is by Connes [4] Lemma 2.1.4 strongly dense in $\mathcal{M}$.

1.3. Connes' compact group action associated to an almost periodic weight (Connes $[6])$.

Definition 1.3.1. (Connes [4]). A normal, faithful, semifinite (n.f.s.) weight $\varphi$ on a von Neumann algebra $\mathcal{M}$ is almost periodic if the modular operator $\Delta_{\varphi}$ on $L^{2}(\mathcal{M}, \varphi)$ is diagonalizable, i.e., the set of eigenvectors of $\Delta_{\varphi}$ has dense linear span in $L^{2}(\mathcal{M}, \varphi)$.

In this paper, when we say $\varphi$ is almost periodic we will always exclude the trivial case when the point spectrum of $\Delta_{\varphi}$ is $\{1\}$, i.e., when $\varphi$ is a trace.

Let $\varphi$ be an almost periodic n.f.s. weight on a von Neumann algebra $\mathcal{M}$. Let $\Gamma \subseteq \mathbf{R}_{+}^{*}$ be the subgroup generated by the point spectrum of $\Delta_{\varphi}$ and endowed with the discrete topology. Let $\hat{\imath}: \Gamma \hookrightarrow \mathbf{R}_{+}^{*}$ denote the inclusion map. Let $G$ be the compact abelian group whose dual is $\Gamma$, with pairing denoted as in Section 1.2. Also, consider $\mathbf{R}_{+}^{*}$ to be the dual of $\mathbf{R}$ under the pairing $\langle t, \mu\rangle=\mu^{i t}$ for $t \in \mathbf{R}, \mu \in \mathbf{R}_{+}^{*}$. Then there is a group homomorphism $\iota: \mathbf{R} \rightarrow G$ determined by $\langle\iota(t), \gamma\rangle=\langle t, \hat{\imath}(\gamma)\rangle \forall \gamma \in G, t \in \mathbf{R}$. Note since $\hat{\iota}$ is injective that the image of $\iota$ is dense in $G$ and also that $\iota$ is injective if and only if $\Gamma$ is dense in $\mathbf{R}_{+}^{*}$.

Lemma 1.3.2. (Connes [6], Proposition 1.1). There is an action $\alpha$ of $G$ on $\mathcal{M}$ such that

(i) $\varphi \circ \alpha_{g}=\varphi \forall g \in G$

(ii) $\alpha_{\iota(t)}=\sigma_{t}^{\varphi} \forall t \in \mathbf{R}$,

where $\sigma^{\varphi}$ is the modular automorphism group associated to $\varphi$. The action above is thus given by

$$
\alpha_{g}(a)=\langle g, \hat{\imath}(\gamma)\rangle a \quad \forall a \in \mathcal{M}_{\varphi}(\gamma)=\mathcal{M}^{\alpha}(\gamma) .
$$

The following lemma is standard and well known. 
Lemma 1.3.3. Let $a \in \mathcal{M}$ and $\gamma \in \Gamma$. Then a belongs to the spectral subspace $\mathcal{M}^{\alpha}(\gamma)$ if and only if

$$
\varphi(b a)=\hat{\imath}(\gamma) \varphi(a b) \forall b \in \mathcal{M}
$$

Proof. This can be proved using the KMS condition exactly like in Takesaki [14] Lemma 1.6, or see Connes [4] Lemma 3.7.5.

The discrete decomposition. In this section, we describe the discrete decomposition of a purely infinite von Neumann algebra with respect to an almost periodic weight, implicit in Connes [6], we develop a useful picture of the $\mathrm{II}_{\infty}$ core of this decomposition, and we show that factoriality of our original algebra is equivallent to ergodicity of a certain partial action of the discrete group on the center of the core.

Let $\mathcal{M}$ be a purely infinite von Neumann algebra and $\varphi$ be a n.f.s. almost periodic weight on $\mathcal{M}$. Let $\alpha$ be Connes' action of the compact group $G$ on $\mathcal{M}$ and $\Gamma=\hat{G}$ as in Section 1.3.

Definition 2.1. We have by Takesaki duality that $\mathcal{M} \simeq\left(\mathcal{M} \rtimes_{\alpha} G\right) \rtimes_{\hat{\alpha}} \Gamma$. The dynamical system $\left(\mathcal{M} \rtimes_{\alpha} G, \Gamma, \hat{\alpha}\right)$ is the discrete decomposition associated to $\varphi$ whose discrete core is $\mathcal{M} \rtimes_{\alpha} G$.

This section is devoted to elucidating the von Neumann algebra $\mathcal{M} \rtimes_{\alpha} G$ and the action $\hat{\alpha}$. We will see that the core of the discrete decomposition is semifinite and the action $\hat{\alpha}$ is trace-scaling (for the embedding of $\Gamma$ in $\mathbf{R}_{+}^{*}$ ). The following proposition gives a useful picture of the discrete core.

Proposition 2.2. Let $\alpha$ be an action of a compact group $G$ on a von Neumann algebra $\mathcal{M} \subseteq B(\mathcal{H})$ and let $\Gamma$ be the dual group of $G$. Then the FourierPlancherel transform provides an isomorphism from $\mathcal{M} \rtimes_{\alpha} G$ onto the von Neumann algebra, $\mathcal{Q}$, acting on $\ell^{2}(\Gamma) \otimes \mathcal{H}$ that is generated by

$$
\left\{M_{f} \otimes 1 \mid f \in l^{\infty}(\Gamma)\right\} \cup\left\{\lambda_{\gamma} \otimes a \mid \gamma \in \Gamma, a \in \mathcal{M}^{\alpha}(\gamma)\right\}
$$

(where $M_{f}$ is the multiplication operator $\left(M_{f} \xi\right)(\gamma)=f(\gamma) \xi(\gamma)$ and $\left(\lambda_{\gamma} \xi\right)\left(\gamma^{\prime}\right)=$ $\xi\left(\gamma^{-1} \gamma^{\prime}\right)$ for $\left.\xi \in \ell^{2}(\Gamma)\right)$. Under this isomorphism the dual automorphism $\hat{\alpha}_{\gamma}$ on $\mathcal{M} \rtimes_{\alpha} G$ corresponds to the automorphism $\operatorname{Ad}\left(\lambda_{\gamma^{-1}} \otimes 1\right)$ on $\mathcal{Q}$. 
Proof. By the definition of crossed product (see Section 1.1) and by Lemma 1.2.3, $\mathcal{M} \rtimes_{\alpha} G$ is isomorphic to the von Neumann algebra on $L^{2}(G) \otimes B(\mathcal{H})$ that is generated by

$$
\left\{\lambda_{g} \otimes 1 \mid g \in G\right\} \cup\left\{\pi_{\alpha, i d}(a) \mid \gamma \in \Gamma, a \in \mathcal{M}^{\alpha}(\gamma)\right\} .
$$

But for $a \in \mathcal{M}^{\alpha}(\gamma)$, since then $\alpha_{h^{-1}}(a)=\langle h, \gamma\rangle a$, we have

$$
\pi_{\alpha, i d}(a)=M_{\langle\cdot, \gamma\rangle} \otimes a,
$$

where $\langle\cdot, \gamma\rangle$ is the function $G \ni h \mapsto\langle h, \gamma\rangle$. Let $\mathcal{F}: L^{2}(G) \rightarrow \ell^{2}(\Gamma)$ be the Fourier-Plancherel transform given by $(\mathcal{F} \xi)(\gamma)=\int_{G} \overline{\langle g, \gamma\rangle} \xi(g) d g \forall \gamma \in \Gamma$. We will conjugate the operators in (4) by $\mathcal{F} \otimes 1$. Now $\mathcal{F} M_{\langle\cdot, \gamma\rangle} \mathcal{F}^{-1}=\lambda_{\gamma}$ and $\mathcal{F} \lambda_{g} \mathcal{F}^{-1}=$ $M_{\overline{\langle g, \cdot\rangle}}$, so $(\mathcal{F} \otimes i d)\left(\mathcal{M} \rtimes_{\alpha} G\right)(\mathcal{F} \otimes i d)^{-1}$ is the von Neumann algebra on $\ell^{2}(\Gamma) \otimes \mathcal{H}$ generated by

$$
\left\{M_{\overline{\langle g, \cdot\rangle}} \otimes 1 \mid g \in G\right\} \cup\left\{\lambda_{\gamma} \otimes a \mid \gamma \in \Gamma, a \in \mathcal{M}^{\alpha}(\gamma)\right\} .
$$

But $\{\overline{\langle g, \cdot\rangle} \mid g \in G\}$ generates $l^{\infty}(\Gamma)$, so conjugation by $\mathcal{F} \otimes i d$ takes $\mathcal{M} \rtimes_{\alpha} G$ onto $\mathcal{Q}$.

The dual automorphism $\hat{\alpha}_{\gamma}$ on the von Neumann algebra generated by the set (4) is $\hat{\alpha}_{\gamma}=\operatorname{Ad}(M \overline{\langle\cdot, \gamma\rangle} \otimes 1)$. But $\mathcal{F} M \overline{\overline{\langle\cdot, \gamma\rangle}} \mathcal{F}^{-1}=\lambda_{\gamma^{-1}}$, proving the last sentence of the proposition.

Remark 2.3. $\mathcal{Q} \subseteq B\left(\ell^{2}(\Gamma)\right) \otimes \mathcal{M}$, and every element of $x \in B\left(\ell^{2}(\Gamma)\right) \otimes \mathcal{M}$ can be viewed as a generalized matrix, indexed over $\Gamma$ and having entries in $\mathcal{M}$, where the $\gamma_{1}, \gamma_{2}$ entry $[x]_{\gamma_{1}, \gamma_{2}}$ is given by

$$
\left\langle[x]_{\gamma_{1}, \gamma_{2}} v, w\right\rangle_{\mathcal{H}}=\left\langle x\left(\chi_{\left\{\gamma_{2}\right\}} \otimes v\right), \chi_{\left\{\gamma_{1}\right\}} \otimes w\right\rangle_{\ell^{2}(\Gamma) \otimes \mathcal{H}}, \forall v, w \in \mathcal{H},
$$

where $\chi_{\left\{\gamma_{i}\right\}} \in \ell^{2}(\Gamma)$ is the characteristic function of $\left\{\gamma_{i}\right\}$. One can easily prove that $\mathcal{Q}$ is the set of all elements $x \in B\left(\ell^{2}(\Gamma)\right) \otimes \mathcal{M}$ that, when viewed as generalized matrices, satisfy $[x]_{\gamma_{1}, \gamma_{2}} \in \mathcal{M}^{\alpha}\left(\gamma_{2}^{-1} \gamma_{1}\right) \forall \gamma_{1}, \gamma_{2} \in \Gamma$. One also checks that

$$
\left[\operatorname{Ad}\left(\lambda_{\gamma^{-1}} \otimes 1\right) x\right]_{\gamma_{1}, \gamma_{2}}=\left[\left(\lambda_{\gamma^{-1}} \otimes 1\right) x\left(\lambda_{\gamma} \otimes 1\right)\right]_{\gamma_{1}, \gamma_{2}}=[x]_{\gamma \gamma_{1}, \gamma \gamma_{2}} .
$$

Thus the discrete action $\hat{\alpha}$ on the discrete core $\mathcal{Q}$ is given by a permution matrix.

Proposition 2.4. Let $\mathcal{M} \subseteq B(\mathcal{H})$ be a von Neumann algebra and $\varphi$ a n.f.s. almost periodic weight on $\mathcal{M}$. Let $G, \alpha$ and $\Gamma$ be as in Section 1.3, and let $\mathcal{Q}$ be as in Proposition 2.2. Then there is a n.f.s. trace $\operatorname{Tr}_{\mathcal{Q}}$ on $\mathcal{Q}$ such that

$$
\operatorname{Tr}_{\mathcal{Q}} \circ \hat{\alpha}_{\gamma}=\hat{\imath}(\gamma)^{-1} \operatorname{Tr}_{\mathcal{Q}}
$$


Proof. Since $\mathcal{Q} \subseteq B\left(\ell^{2}(\Gamma)\right) \otimes \mathcal{M}$ let $\operatorname{Tr}_{\mathcal{Q}}$ be $\operatorname{Tr}\left(M_{\hat{\imath}} \cdot\right) \otimes \varphi$ restricted to $\mathcal{Q}$, where $\operatorname{Tr}$ is the n.f.s. trace on $B\left(\ell^{2}(\Gamma)\right)$ and the density matrix $M_{\hat{\imath}}$ is the multiplication operator associated to the unbounded positive function $\hat{\imath}$ on $\Gamma$. Then $\operatorname{Tr}_{\mathcal{Q}}$ is normal and semifinite because $\operatorname{Tr}$ and $\varphi$ are n.f.s. and because $M_{\hat{\iota}}$ is affiliated to $\mathcal{Q}$. To see that $\operatorname{Tr}_{\mathcal{Q}}$ is a trace, let $\mathfrak{m}=\left\{a \in \mathcal{M} \mid \varphi\left(a^{*} a\right)<+\infty\right\}$. Then there is a net of projections $e_{i} \in \mathcal{M}^{\alpha},(i \in I)$, increasing to one, which together with Lemma 1.2.3 shows that $\operatorname{span} \bigcup_{\gamma \in \Gamma}\left(\mathcal{M}^{\alpha}(\gamma) \cap \mathfrak{m}\right)$ is a strongly dense *-subalgebra of $\mathcal{M}$. Hence it will suffice to show that $\operatorname{Tr}_{\mathcal{Q}}(x y)=\operatorname{Tr}_{\mathcal{Q}}(y x)$ whenever $x$ and $y$ are finite products of elements in

\section{$\left\{M_{f} \mid f \in l^{\infty}(\Gamma)\right.$ having finite support $\} \cup\left\{\lambda_{\gamma} \otimes a \mid \gamma \in \Gamma, a \in \mathcal{M}^{\alpha}(\gamma) \cap \mathfrak{m}\right\}$.}

But such a finite product is equal to $\left(M_{f} \otimes 1\right)\left(\lambda_{\gamma} \otimes a\right)$ for some $f \in l^{\infty}(\Gamma)$ having finite support, some $\gamma \in \Gamma$ and $a \in \mathcal{M}^{\alpha}(\gamma)$. This in turn is the sum of operators of the form $\left(\chi_{\gamma^{\prime}} \otimes 1\right)\left(\lambda_{\gamma} \otimes a\right)$, which when viewed as a generalized matrix as in Remark 2.3 has all entries equal to zero except the $\gamma^{\prime}, \gamma^{\prime} \gamma^{-1}$ entry. Hence it suffices to show that $\operatorname{Tr}_{\mathcal{Q}}(x y)=\operatorname{Tr}_{\mathcal{Q}}(y x)$ when $[x]_{\gamma_{1}, \gamma_{2}}=a$ and all other entries of $x$ are zero and $[y]_{\gamma_{3}, \gamma_{4}}=b$ and all other entries of $y$ are zero. Both $\operatorname{Tr}_{\mathcal{Q}}(x y)$ and $\operatorname{Tr}_{\mathcal{Q}}(y x)$ are zero unless $\gamma_{3}=\gamma_{2}$ and $\gamma_{4}=\gamma_{1}$, so assume this is the case. Then $a \in \mathcal{M}^{\alpha}\left(\gamma_{2}^{-1} \gamma_{1}\right)$ and $b \in \mathcal{M}^{\alpha}\left(\gamma_{1}^{-1} \gamma_{2}\right)$, so by Lemma 1.3.3,

$$
\operatorname{Tr}_{\mathcal{Q}}(y x)=\hat{\imath}\left(\gamma_{2}\right) \varphi(b a)=\hat{\iota}\left(\gamma_{1}\right) \varphi(a b)=\operatorname{Tr}_{\mathcal{Q}}(x y) .
$$

We know exhibit a partial action, $S_{\gamma}$, of $\Gamma$ on the center of the centralizer of $\mathcal{M}$, and show that factoriality of $\mathcal{M}$ is equivalent to ergodicity of this partial action. Let us first take a closer look at spectral subspaces $\mathcal{M}^{\alpha}(\gamma)$. Taking $\mathcal{M}$, $\varphi$, etc. as in the previous proposition, by Lemma 1.3.3 $\mathcal{M}^{\alpha}$ equals the centralizer, $\mathcal{M}_{\varphi}$, of $\varphi$. Thus for $\gamma \in \Gamma$, we will also denote the spectral subspace $\mathcal{M}^{\alpha}(\gamma)$ by $\mathcal{M}_{\varphi}(\gamma)$. Let $Z_{\varphi}=Z\left(\mathcal{M}_{\varphi}\right)$ denote the center of the centralizer of $\varphi$ and $P Z_{\varphi}$ the set of (self-adjoint) projections in $Z_{\varphi}$. In general, for $a$ an element in a von Neumann algebra $\mathcal{N}, C_{\mathcal{N}}(a)$ will denote the central carrier of $a$ in $\mathcal{N}$.

Definition 2.5. For $\gamma \in \Gamma$ and $p \in P Z_{\varphi}$ let

$$
S_{\gamma}(p)=\bigvee\left\{C_{\mathcal{M}_{\varphi}}\left(a p a^{*}\right) \mid a \in \mathcal{M}_{\varphi}(\gamma)\right\} \in P Z_{\varphi}
$$

\section{Lemma 2.6.}

(i) $\forall \gamma \in \Gamma$ there is a partial isometry $v_{\gamma} \in \mathcal{M}_{\varphi}(\gamma)$ such that

$$
S_{\gamma}(p)=C_{\mathcal{M}_{\varphi}}\left(v_{\gamma} p v_{\gamma}^{*}\right) \forall p \in P Z_{\varphi} ;
$$


(ii) one may arrange that $v_{\gamma^{-1}}=v_{\gamma}^{*}$.

(iii) Let $\gamma \in \Gamma$; then $p \leq p^{\prime} \Rightarrow S_{\gamma}(p) \leq S_{\gamma}\left(p^{\prime}\right) \forall p, p^{\prime} \in P Z_{\varphi}$;

(iv) setting $p_{\gamma}=S_{\gamma^{-1}}(1)$ we have $S_{\gamma}(p)=0$ if $p \perp p_{\gamma}$ and, for any $p \in P Z_{\varphi}$, $S_{\gamma^{-1}} S_{\gamma}(p)=p p_{\gamma}$

(v) if $p_{1}, p_{2} \in p_{\gamma} P Z_{\varphi}$ then $S_{\gamma}\left(p_{1}\right)=S_{\gamma}\left(p_{2}\right)$ implies $p_{1}=p_{2}$;

(vi) $S_{\gamma}\left(p_{1} p_{2}\right)=S_{\gamma}\left(p_{1}\right) S_{\gamma}\left(p_{2}\right) \forall p_{1}, p_{2} \in P Z_{\varphi}$;

(vii) if $p_{1}, p_{2} \in P Z_{\varphi}$ and $p_{1} \perp p_{2}$ then $S_{\gamma}\left(p_{1}+p_{2}\right)=S_{\gamma}\left(p_{1}\right)+S_{\gamma}\left(p_{2}\right)$.

(viii) $S_{\gamma}$ is normal in the sense that if $\left(p_{n}\right)_{n=1}^{\infty}$ is an increasing family of projections in $Z_{\varphi}$ then

$$
S_{\gamma}\left(\bigvee_{n=1}^{\infty} p_{n}\right)=\bigvee_{n=1}^{\infty} S_{\gamma}\left(p_{n}\right)
$$

Proof. For (i), let $\left(v_{i}\right)_{i \in I}$ be a family of partial isometries in $\mathcal{M}_{\varphi}(\gamma)$ that is maximal with respect to the property that $C_{\mathcal{M}_{\varphi}}\left(v_{i}^{*} v_{i}\right) \perp C_{\mathcal{M}_{\varphi}}\left(v_{i^{\prime}}^{*} v_{i^{\prime}}\right)$ and $C_{\mathcal{M}_{\varphi}}\left(v_{i} v_{i}^{*}\right) \perp C_{\mathcal{M}_{\varphi}}\left(v_{i^{\prime}} v_{i^{\prime}}^{*}\right)$ whenever $i, i^{\prime} \in I, i \neq i^{\prime}$. Let $v_{\gamma}=\sum_{i \in I} v_{i}$. For $p \in P Z_{\varphi}$, let $q=C_{\mathcal{M}_{\varphi}}\left(v_{\gamma} p v_{\gamma}^{*}\right)$. Clearly $q \leq S_{\gamma}(p)$. Suppose for contradiction that $q \neq S_{\gamma}(p)$. Then $\exists a \in \mathcal{M}_{\varphi}(\gamma)$ such that $(1-q) a p \neq 0$. Taking the polar decomposition, let $w$ be the polar part of $(1-q) a p \neq 0$. Then $w \in \mathcal{M}_{\varphi}(\gamma)$ is a partial isometry, $w^{*} w \leq p$ and $w w^{*} \leq(1-q)$. We will show that $C_{\mathcal{M}_{\varphi}}\left(w w^{*}\right) \perp C_{\mathcal{M}_{\varphi}}\left(v_{i} v_{i}^{*}\right)$ and $C_{\mathcal{M}_{\varphi}}\left(w^{*} w\right) \perp C_{\mathcal{M}_{\varphi}}\left(v_{i}^{*} v_{i}\right) \forall i \in I$, which will contradict the maximality of $\left(v_{i}\right)_{i \in I}$. If for some $i \in I, C_{\mathcal{M}_{\varphi}}\left(w w^{*}\right) C_{\mathcal{M}_{\varphi}}\left(v_{i} v_{i}^{*}\right) \neq 0$ then $\exists a \in \mathcal{M}_{\varphi}$ such that $w^{*} a v_{i} \neq 0$. But $w^{*} a v_{i} \in \mathcal{M}_{\varphi}$ so $0 \neq p w^{*}(1-q) a v_{i}=$ $w^{*} a(1-q) v_{i} p$ and $(1-q) v_{i} p \neq 0$, contradicting the choice of $q$. Similarly, if if $C_{\mathcal{M}_{\varphi}}\left(w^{*} w\right) C_{\mathcal{M}_{\varphi}}\left(v_{i}^{*} v_{i}\right) \neq 0$ then $\exists a \in \mathcal{M}_{\varphi}$ such that $w a v_{i}^{*} \neq 0$, so $0 \neq$ $(1-q)$ wpav $_{i}^{*}=\operatorname{wapv}_{i}^{*}(1-q)$, a contradiction.

For (ii), just note from the proof of (i) that any maximal family $\left(v_{i}\right)_{i \in I}$ will do, and use that $\mathcal{M}_{\varphi}\left(\gamma^{-1}\right)=\mathcal{M}_{\varphi}(\gamma)^{*}$.

Part (iii) is clear from the definition of $S_{\gamma}$. For (iv), we have from (i) and (ii) that $p_{\gamma}=C_{\mathcal{M}_{\varphi}}\left(v_{\gamma}^{*} v_{\gamma}\right)$. If $p \in P Z_{\varphi}$ and $p \perp p_{\gamma}$, then $p \perp v_{\gamma}^{*} v_{\gamma}$, so $S_{\gamma}(p)=C_{\mathcal{M}_{\varphi}}\left(v_{\gamma} p v_{\gamma}^{*}\right)=0$. For any $p \in P Z_{\varphi}, S_{\gamma}(p) \geq v_{\gamma} p v_{\gamma}^{*}$ so $S_{\gamma^{-1}} S_{\gamma}(p)=$ $C_{\mathcal{M}_{\varphi}}\left(v_{\gamma}^{*} S_{\gamma}(p) v_{\gamma}\right) \geq C_{\mathcal{M}_{\varphi}}\left(p v_{\gamma}^{*} v_{\gamma}\right)=p p_{\gamma}$. But also

$$
\begin{aligned}
S_{\gamma^{-1}} S_{\gamma}(p) & =C_{\mathcal{M}_{\varphi}}\left(v_{\gamma}^{*} S_{\gamma}(p) v_{\gamma}\right) \\
& =C_{\mathcal{M}_{\varphi}}\left(v_{\gamma}^{*} C_{\mathcal{M}_{\varphi}}\left(v_{\gamma} p v_{\gamma}^{*}\right) v_{\gamma}\right) \\
& \leq C_{\mathcal{M}_{\varphi}}\left(v_{\gamma}^{*} v_{\gamma} p v_{\gamma}^{*} v_{\gamma}\right)=p p_{\gamma},
\end{aligned}
$$

proving (iv). Part (v) is clear from (iv). 
For (vi), $\begin{aligned} S_{\gamma}\left(p_{1} p_{2}\right) \leq & S_{\gamma}\left(p_{1}\right) S_{\gamma}\left(p_{2}\right) \text { from (iii). Using (iv), } \\ p_{\gamma} p_{1} p_{2} & =S_{\gamma^{-1}} S_{\gamma}\left(p_{1} p_{2}\right) \leq S_{\gamma^{-1}}\left(S_{\gamma}\left(p_{1}\right) S_{\gamma}\left(p_{2}\right)\right) \\ & \leq\left(S_{\gamma^{-1}} S_{\gamma}\left(p_{1}\right)\right)\left(S_{\gamma^{-1}} S_{\gamma}\left(p_{2}\right)\right)=p_{\gamma} p_{1} p_{2} .\end{aligned}$

Hence the inequalities in the previous sentence are equalities, so by (v), $S_{\gamma}\left(p_{1} p_{2}\right)$ $=S_{\gamma}\left(p_{1}\right) S_{\gamma}\left(p_{2}\right)$. hence

For (vii), we have from (vi) and (i) that $C_{\mathcal{M}_{\varphi}}\left(v_{\gamma} p_{1} v_{\gamma}^{*}\right) \perp C_{\mathcal{M}_{\varphi}}\left(v_{\gamma} p_{2} v_{\gamma}^{*}\right)$,

$$
\begin{aligned}
S_{\gamma}\left(p_{1}+p_{2}\right) & =C_{\mathcal{M}_{\varphi}}\left(v_{\gamma} p_{1} v_{\gamma}^{*}+v_{\gamma} p_{2} v_{\gamma}^{*}\right) \\
& =C_{\mathcal{M}_{\varphi}}\left(v_{\gamma} p_{1} v_{\gamma}^{*}\right)+C_{\mathcal{M}_{\varphi}}\left(v_{\gamma} p_{2} v_{\gamma}^{*}\right) S_{\gamma}\left(p_{1}\right)+S_{\gamma}\left(p_{2}\right) .
\end{aligned}
$$

Part (viii) holds because taking central carriers respects $\bigvee$ (see Kadison and Ringrose [11], 5.5.3).

\section{Lemma 2.7.}

(i) $S_{\gamma}$ extends to a normal $*-$ homomorphism, also denoted $S_{\gamma}$, from $Z_{\varphi}$ into $Z_{\varphi}$; the kernel of $S_{\gamma}$ is $\left(1-p_{\gamma}\right) Z_{\varphi}$ and the restriction of $S_{\gamma}$ is an isomorphism from $p_{\gamma} Z_{\varphi}$ onto $p_{\gamma^{-1}} Z_{\varphi}$.

(ii) We have $p_{1_{\Gamma}}=1$ and $S_{1_{\Gamma}}=i d$.

(iii) Let $\gamma_{1}, \gamma_{2} \in \Gamma$ and $q=S_{\gamma_{2}^{-1}}\left(p_{\gamma_{1}}\right)$. Then $q \leq p_{\gamma_{1} \gamma_{2}}$ and

$$
\left.S_{\gamma_{1}} S_{\gamma_{2}}\right|_{q Z_{\varphi}}=\left.S_{\gamma_{1} \gamma_{2}}\right|_{q Z_{\varphi}}
$$

Proof. For (i), the properties proved in Lemma 2.6 show that $S_{\gamma}$ extends to a homomorphism from $\operatorname{span} P Z_{\varphi}$ (i.e., the $L^{\infty}$ functions taking only finitely many values) to $\operatorname{span} P Z_{\varphi}$, with kernel $\operatorname{span}\left(1-p_{\gamma}\right) P Z_{\varphi}$ and that is $1-1$ on span $p_{\gamma} P Z_{\varphi}$. Extending to all of $P Z_{\varphi}$ is now standard measure theory, making use of part (viii) of Lemma 2.6. Part (ii) is clear.

For (iii), let us show that

$$
S_{\gamma_{1}} S_{\gamma_{2}}(p)=S_{\gamma_{1} \gamma_{2}}(q p) \forall p \in P Z_{\varphi} .
$$

Denoting by $\operatorname{supp}(b)$ the support in $\mathcal{M}_{\varphi}$ of a self-adjoint element $b \in \mathcal{M}_{\varphi}$, we have

$$
\begin{aligned}
S_{\gamma_{1}} S_{\gamma_{2}}(p) & =\bigvee_{a_{1} \in \mathcal{M}_{\varphi}\left(\gamma_{1}\right)} \operatorname{supp}\left(a_{1}\left(\bigvee_{a_{2} \in \mathcal{M}_{\varphi}\left(\gamma_{2}\right)} \operatorname{supp}\left(a_{2} p a_{2}^{*}\right)\right) a_{1}^{*}\right) \\
& \leq \bigvee_{\substack{a_{1} \in \mathcal{M}_{\varphi}\left(\gamma_{1}\right) \\
a_{2} \in \mathcal{M}_{\varphi}\left(\gamma_{2}\right)}} a_{1} a_{2} p a_{2}^{*} a_{1}^{*} \\
& \leq \bigvee_{a \in \mathcal{M}_{\varphi}\left(\gamma_{1} \gamma_{2}\right)} a p a^{*}=S_{\gamma_{1} \gamma_{2}}(p)
\end{aligned}
$$


Hence

$$
S_{\gamma_{1}} S_{\gamma_{2}}(p)=S_{\gamma_{1}}\left(p_{\gamma_{1}} S_{\gamma_{2}}(p)\right)=S_{\gamma_{1}}\left(p_{\gamma_{1}} p_{\gamma_{2}^{-1}} S_{\gamma_{2}}(p)\right)=S_{\gamma_{1}} S_{\gamma_{2}}(q p) \leq S_{\gamma_{1} \gamma_{2}}(q p) .
$$

But $q p=S_{\gamma_{2}^{-1}}\left(p_{\gamma_{1}} S_{\gamma_{2}}(p)\right)$ so

$$
S_{\gamma_{1} \gamma_{2}}(q p) \leq S_{\gamma_{1}}\left(p_{\gamma_{1}} S_{\gamma_{2}}(p)\right)=S_{\gamma_{1}} S_{\gamma_{2}}(p)
$$

which proves (5).

Now it only remains to show that $q \leq p_{\gamma_{1} \gamma_{2}}$. For this it will suffice by part (i) to show that $p \in P Z_{\varphi}, p \leq q$ and $S_{\gamma_{1} \gamma_{2}}(p)=0$ implies $p=0$. Then by (5), the assumptions imply $S_{\gamma_{1}} S_{\gamma_{2}}(p)=0$, so by part (i) $S_{\gamma_{2}}(p) \perp p_{\gamma_{1}}$. Hence (because $\left.p \leq q \leq p_{\gamma_{2}}\right)$ by Lemma 2.6iv, $p=S_{\gamma_{2}^{-1}} S_{\gamma_{2}}(p) \perp S_{\gamma_{2}^{-1}}\left(p_{\gamma_{1}}\right)=q$, so $p=0$.

Remark 2.8. From the lemmas above we see by standard arguments that $Z_{\varphi}=L^{\infty}(X, \mu)$ where $X$ is a standard Borel space with measure $\mu$ and for each $\gamma \in \Gamma, p_{\gamma}$ is the characteristic function of $A_{\gamma} \subseteq X$ and $S_{\gamma}(f)=f \circ T_{\gamma^{-1}}$ where $T_{\gamma^{-1}}: A_{\gamma^{-1}} \rightarrow A_{\gamma}$ is a bijective measurable mapping. Moreover, $T_{\gamma^{-1}}=\left(T_{\gamma}\right)^{-1}$ and $\left.T_{\gamma_{2}} \circ T_{\gamma_{1}}\right|_{B}=\left.T_{\gamma_{2} \gamma_{1}}\right|_{B}$ where $B=T_{\gamma_{1}^{-1}}\left(A_{\gamma_{1}^{-1}} \cap A_{\gamma_{2}}\right) \subseteq A_{\gamma_{1} \gamma_{2}}$.

Thus we can describe $\Gamma \ni \gamma \mapsto T_{\gamma}$ as a partial action of $\Gamma$ on $X$. Let us define a measurable subset $F \subseteq X$ to be invariant under the partial action $T$ if $T_{\gamma}\left(F \cap A_{\gamma}\right)=F \cap A_{\gamma^{-1}} \forall \gamma \in \Gamma$, and the partial action $T$ to be ergodic if every invariant subset is null or conull. An equivalent condition for the ergodicity of $T$ is in terms of measurable equivalence relations of Feldman and Moore [9]: the union of the graphs of $T_{\gamma}$ as $\gamma$ ranges over all of $\Gamma$ is a measurable equivalence relation. Then $T$ is ergodic if and only if the equivalence relation is ergodic.

The following proposition says that $T$ is ergodic if and only if $\mathcal{M}$ is a factor; but in order to avoid using the techniques of measure theory, the condition of ergodicity is expressed in terms of the maps $S_{\gamma}$.

Proposition 2.9. Let $x \in \mathcal{M}$. Then $x$ is in the center of $\mathcal{M}$ if and only if $x$ is in the center of $\mathcal{M}_{\varphi}$ and

$$
S_{\gamma}(x)=x p_{\gamma^{-1}} \forall \gamma \in \Gamma .
$$

Proof. Since both $Z(\mathcal{M})$ and the set of $x \in \mathcal{M}$ satisfying (6) are von Neumann subalgebras of $\mathcal{M}$, we may assume $x$ is a projection in $\mathcal{M}$. To show necessity, suppose $p \in P Z(\mathcal{M})$. Then $p \in P Z_{\varphi}$. For all $\gamma \in \Gamma$ we have

$$
\begin{aligned}
S_{\gamma}(p) & =\bigvee\left\{C_{\mathcal{M}_{\varphi}}\left(a p a^{*}\right) \mid a \in \mathcal{M}_{\varphi}(\gamma)\right\} \\
& =\bigvee\left\{p C_{\mathcal{M}_{\varphi}}\left(a a^{*}\right) \mid a \in \mathcal{M}_{\varphi}(\gamma)\right\}=p p_{\gamma^{-1}}
\end{aligned}
$$

To show sufficiency, let $x=p \in P Z_{\varphi}$ such that (6) holds. To show $p \in Z(\mathcal{M})$ it will by Lemma 1.2.3 suffice to show that $p$ commutes with $a$ for every $\gamma \in \Gamma$ and $a \in \mathcal{M}_{\varphi}(\gamma)$. But if not then $(1-p) a p \neq 0$, so $(1-p) C_{\mathcal{M}_{\varphi}}\left(a p a^{*}\right) \neq 0$, so $(1-p) S_{\gamma}(p) \neq 0$, contradicting $S_{\gamma}(p)=p p_{\gamma^{-1}}$. 
Corollary 2.10. $\mathcal{M}$ is a factor if and only if the partial action $T$ is ergodic.

Proposition 2.11. Let $\mathcal{M}, \varphi, G, \alpha, \Gamma$ and $\mathcal{Q} \simeq \mathcal{M} \rtimes_{\alpha} G$ be as in Proposition 2.4, and regard elements of $\mathcal{Q}$ as generalized matrices as described in Remark 2.3. Let $x \in \mathcal{Q}$. Then $x$ is in the center of $\mathcal{Q}$ if and only if

(1) $[x]_{\gamma_{1}, \gamma_{2}}=0$ whenever $\gamma_{1}, \gamma_{2} \in \Gamma, \gamma_{1} \neq \gamma_{2}$;

(2) $[x]_{\gamma, \gamma} \in Z_{\varphi} \forall \gamma \in \Gamma$; and

(3) $S_{\gamma_{1}}\left([x]_{\gamma, \gamma} p_{\gamma_{1}}\right)=[x]_{\gamma \gamma_{1}, \gamma \gamma_{1}} p_{\gamma_{1}^{-1}} \forall \gamma, \gamma_{1} \in \Gamma$.

Proof. The set of $x \in \mathcal{Q}$ satisfying (1)-(3) is a von Neumann subalgebra of $\mathcal{Q}$, so we may assume $x=q$ is a projection in $\mathcal{Q}$. To prove necessity, suppose $q \in$ $Z(\mathcal{Q})$. Then since every bounded, diagonal operator having entries in $\mathcal{M}_{\varphi}$ is in $\mathcal{Q}$, clearly (1) and (2) hold. Suppose for contradiction that (3) fails for some $\gamma_{1}, \gamma_{2} \in$ $\Gamma$. We may assume that $\left(1-[q]_{\gamma \gamma_{1}, \gamma \gamma_{1}}\right) S_{\gamma_{1}}\left([q]_{\gamma, \gamma} p_{\gamma_{1}}\right) \neq 0$, (otherwise take $S_{\gamma_{1}^{-1}}$ of both sides). Hence $\exists a \in \mathcal{M}_{\varphi}\left(\gamma_{1}\right)$ such that $a[q]_{\gamma, \gamma}=a \neq 0$ and $a a^{*}=$ $a[q]_{\gamma, \gamma} a^{*} \perp[q]_{\gamma \gamma_{1}, \gamma \gamma_{1}}$. Let $y \in \mathcal{Q}$ have entry $[y]_{\gamma \gamma_{1}, \gamma}=a$ and all other entries equal to 0 . It follows that $[y q]_{\gamma \gamma_{1}, \gamma}=a[q]_{\gamma, \gamma} \neq 0$ and $[q y]_{\gamma \gamma_{1}, \gamma}=[q]_{\gamma \gamma_{1}, \gamma \gamma_{1}} a=0$, contradicting that $q \in Z(\mathcal{Q})$.

For sufficiency, suppose $q \in P \mathcal{Q}$ satisfies (1)-(3). To show $q \in Z(\mathcal{Q})$ it will suffice to show that $y q=q y$ whenever $y \in \mathcal{Q}$ has only one nonzero entry, i.e., to show

$$
a[q]_{\gamma, \gamma}=[q]_{\gamma \gamma_{1}, \gamma \gamma_{1}} a \quad \forall \gamma, \gamma_{1} \in \Gamma \forall a \in \mathcal{M}_{\varphi}\left(\gamma_{1}\right) .
$$

Since $a^{*} a \in \mathcal{M}_{\varphi}$, taking the polar decomposition of $a$, one sees that it suffices to show (7) for $a=v \in \mathcal{M}_{\varphi}\left(\gamma_{1}\right)$ a partial isometry. But $v v^{*} \leq p_{\gamma_{1}^{-1}}$, so using (3) it suffices to show $v[q]_{\gamma, \gamma} v^{*}=S_{\gamma_{1}}\left([q]_{\gamma, \gamma} p_{\gamma}\right) v v^{*}$, hence, since $v^{*} v \leq p_{\gamma}$, to show

$$
v r v^{*}=S_{\gamma_{1}}(r) v v^{*} \forall r \in p_{\gamma_{1}} P Z_{\varphi} \quad \forall \gamma_{1} \in \Gamma
$$

$\forall$ partial isometries $v \in \mathcal{M}_{\varphi}\left(\gamma_{1}\right)$.

But $v r v^{*} \leq S_{\gamma_{1}}(r)$ by definition, so $\leq$ holds in (8). Since $r=S_{\gamma_{1}^{-1}} S_{\gamma_{1}}(r)$ we may apply $\leq$ of (8) to get $v r v^{*}=v\left(v^{*} v S_{\gamma_{1}^{-1}}\left(S_{\gamma_{1}}(r)\right)\right) v^{*} \geq v\left(v^{*} S_{\gamma_{1}}(r) v\right) v^{*}=$ $S_{\gamma_{1}}(r) v v^{*}$.

Proposition 2.12. The discrete core $\mathcal{Q}$ is a factor if and only if $\mathcal{M}_{\varphi}$ is a factor, and in that case $\Gamma$ equals the point spectrum of $\Delta_{\varphi}$ and $\mathcal{Q} \simeq \mathcal{M}_{\varphi} \otimes B(\mathcal{H})$, where $\mathcal{H}$ is separable, infinite dimensional Hilbert space. 
Proof. Suppose that $\mathcal{M}_{\varphi}$ is not a factor, let $p$ be a nontrivial projection in the center of $\mathcal{M}_{\varphi}$ and let $q \in \mathcal{Q}$, satisfying (1)-(3) of the proposition, be such that $[q]_{\gamma, \gamma}=S_{\gamma}(p)$. Then by Proposition 2.11, $q$ is in the center of $\mathcal{Q}$ and $\mathcal{Q}$ is not a factor.

Suppose that $\mathcal{M}_{\varphi}$ is a factor. Then for each $\gamma \in \Gamma, p_{\gamma}=0$ or 1 , and $p_{\gamma}=0$ if and only if $\mathcal{M}_{\varphi}(\gamma)=\{0\}$ if and only if $\gamma$ is not in the point spectrum of $\Delta_{\varphi}$. By Lemma 2.7iii, $\Gamma^{\prime}=\left\{\gamma \in \Gamma \mid p_{\gamma}=1\right\}$ is a subgroup of $\Gamma$. But $\Gamma^{\prime}$ equals the point spectrum of $\Delta_{\varphi}$ and $\Gamma$ was taken to be the group generated by $\Gamma^{\prime}$, so $\Gamma^{\prime}=\Gamma$. Choose any $\gamma_{0} \in \Gamma$ and let $q \in \mathcal{Q}$ be such that $[q]_{\gamma_{0}, \gamma_{0}}=1$ and all other entries of $q$ are zero. Then since $p_{\gamma}=1 \forall \gamma \in \Gamma$, it follows that the central carrier of $q$ in $\mathcal{Q}$ equals the identity of $\mathcal{Q}$. But $q \mathcal{Q} q \simeq \mathcal{M}_{\varphi}$, so $\mathcal{Q}$ is a factor. Looking to Proposition 2.4 and noting that $\Gamma$ is an infinite set, we see that $\mathcal{Q}$ is a type $\mathrm{II}_{\infty}$ factor and $\mathcal{Q} \simeq q \mathcal{Q} q \otimes B(\mathcal{H})$.

3. The continuous decomposition. In this section, for $\mathcal{M}$ a purely infinite von Neumann algebra with n.f.s. almost periodic weight $\varphi$, Takesaki's continuous decomposition for $\mathcal{M}$ (see Section 1.1) is related to the discrete decomposition obtained in Section 2. We shall see that Takesaki's continuous decomposition can be viewed as the induced representation of the discrete decomposition, if one broadens Takesaki's definition of induced representation to include actions induced up from dense subgroups. For this notion of induced action, one must consider a locally compact topology on the subgroup, which may be different from the restriction topology, an example of which is $\mathbf{Q} \subseteq \mathbf{R}$, with the usual separable topology on $\mathbf{R}$ and the discrete topology on $\mathbf{Q}$. What follows is Takesaki's definition [15] of induced action, except that he required $H$ to be a closed subgroup with the restriction topology.

Definition 3.1. Let $K$ be a locally compact abelian group, $H \subseteq K$ a subgroup. Suppose $\alpha$ is an action of $H$ on a von Neumann algebra $\mathcal{N}$, continous with respect to some locally compact topology on $H$ that is finer than the restriction of the topology on $K$. Consider the von Neumann algebra $L^{\infty}(K) \otimes$ $\mathcal{N} \simeq L^{\infty}(K, \mathcal{N})$, equal to the set of bounded measurable functions from $K$ into $\mathcal{N}$, and the action $\beta$ of $H$ on $L^{\infty}(K, \mathcal{N})$ given by $\left(\beta_{h} f\right)(k)=\alpha_{h}(f(k h))$ for $h \in H, f \in L^{\infty}(K, \mathcal{N})$ and $k \in K$. Let $\mathcal{M}$ be the fixed-point subalgebra of $L^{\infty}(K, \mathcal{N})$ under the action $\beta$ of $H$. The action, $\tau$, of $K$ on $L^{\infty}(K, \mathcal{N})$ given by $\left(\tau_{k} f\right)\left(k^{\prime}\right)=f\left(k^{-1} k^{\prime}\right)$ leaves $\mathcal{M}$ globally invariant. The action induced up to $K$ from the action $\alpha$ of $H$ on $\mathcal{N}$ is the action $\tau$ of $K$ restricted to $\mathcal{M}$.

Let $G$ be a compact abelian group with dual group $\Gamma, \alpha$ an action of $G$ on a von Neumann algebra $\mathcal{M}, E$ a locally compact abelian group, $\iota: E \rightarrow G$ a continuous homomorphism whose image is dense in $G$. Then $\alpha \circ \iota$ is an action 
of $E$ on $\mathcal{M}$. Let $\hat{\iota}: \Gamma \rightarrow \hat{E}$ be the injective homomorphism given by $\langle t, \hat{\iota}(\gamma)\rangle=$ $\langle\iota(t), \gamma\rangle \forall t \in E, \gamma \in \Gamma$. Let

$$
\begin{aligned}
& \mathcal{P}=\mathcal{M} \rtimes_{\alpha} G \\
& \widetilde{\mathcal{P}}=\mathcal{M} \rtimes_{\alpha \circ \iota} E .
\end{aligned}
$$

Proposition 3.2. Let $\tilde{\pi}$ be a normal, faithful representation of $\mathcal{M}$ on the Hilbert space $\mathcal{H}$. Then the Fourier-Plancherel transform provides an isomorphism from $\widetilde{\mathcal{P}}$ onto the von Neumann algebra, $\widetilde{\mathcal{Q}}$, acting on $L^{2}(\hat{E}) \otimes \mathcal{H}$, that is generated by

$$
\left\{M_{F} \otimes 1 \mid F \in L^{\infty}(\hat{E})\right\} \cup\left\{\lambda_{\hat{\iota}(\gamma)} \otimes \tilde{\pi}(a) \mid \gamma \in \Gamma, a \in \mathcal{M}^{\alpha}(\gamma)\right\},
$$

(where $M_{F}$ is the multiplication operator on $\left.L^{2}(\hat{E})\right)$. Under this isomorphism, the dual automorphism $(\alpha \circ \iota)_{\mu}^{\wedge}$ on $\widetilde{\mathcal{P}}$ for $\mu \in \hat{E}$ corresponds to $\operatorname{Ad}\left(\lambda_{\mu^{-1}} \otimes 1\right)$ on $\widetilde{\mathcal{Q}}$.

Proof. This proposition is proved just like Proposition 2.2, once one notes that if $a \in \mathcal{M}^{\alpha}(\gamma)$ and $t \in E$ then $(\alpha \circ \iota)_{t^{-1}}(a)=\langle t, \hat{\imath}(\gamma)\rangle a$, so that $\pi_{\alpha \circ \iota, \tilde{\pi}}(a)=$ $M_{\langle\cdot, \hat{\imath}(\gamma)\rangle} \otimes \tilde{\pi}(a)$.

Proposition 3.3. In the situation of Proposition 3.2, let the action of $\hat{E}$ that is induced up from the action $\hat{\alpha}$ of $\Gamma$ on $\mathcal{P}$ be denoted the action $\tau$ of $\hat{E}$ on $\mathcal{R}$. Then there is an isomorphism from $\widetilde{\mathcal{P}}$ onto $\mathcal{R}$ that intertwines the dual action $(\alpha \circ \iota)^{\wedge}$ of $\hat{E}$ on $\widetilde{\mathcal{P}}$ with the action $\tau$.

Proof. Let $\pi_{\alpha, i d}$ be the representation of $\mathcal{M}$ on $L^{2}(G) \otimes \mathcal{H}$ used in the proof of Proposition 2.2 and let $\mathcal{F}: L^{2}(G) \rightarrow \ell^{2}(\Gamma)$ be the Fourier-Plancherel transform. Then $\tilde{\pi}=(\mathcal{F} \otimes 1) \pi_{\alpha, i d}(\mathcal{F} \otimes 1)^{-1}$ is a faithful, normal representation of $\mathcal{M}$ on $\ell^{2}(\Gamma) \otimes \mathcal{H}$ and $\tilde{\pi}(a)=\lambda_{\gamma} \otimes a$ if $\gamma \in \Gamma$ and $a \in \mathcal{M}^{\alpha}(\gamma)$, so by Proposition 3.2, $\widetilde{\mathcal{P}}$ is isomorphic to the von Neumann algebra acting on $L^{2}(\hat{E}) \otimes \ell^{2}(\Gamma) \otimes \mathcal{H}$ that is generated by

$$
\left\{M_{F} \otimes 1 \otimes 1 \mid F \in L^{\infty}(\hat{E})\right\} \cup\left\{\lambda_{\hat{\iota}(\gamma)} \otimes \lambda_{\gamma} \otimes a \mid \gamma \in \Gamma, a \in \mathcal{M}^{\alpha}(\gamma)\right\}
$$

and the dual automorphism $(\alpha \circ \iota)_{\mu}^{\wedge}$ for $\mu \in \hat{E}$ is given by $\operatorname{Ad}\left(\lambda_{\mu^{-1}} \otimes 1 \otimes 1\right)$.

By Proposition $2.2, L^{\infty}(\hat{E}, \mathcal{P})$ is isomorphic to the von Neumann algebra acting on $L^{2}(\hat{E}) \otimes \ell^{2}(\Gamma) \otimes \mathcal{H}$ that is generated by

$$
\left\{M_{\langle t, \cdot\rangle} \otimes 1 \otimes 1 \mid t \in E\right\} \cup\left\{1 \otimes M_{\langle g, \cdot\rangle} \otimes 1 \mid g \in G\right\}
$$

$\cup\left\{1 \otimes \lambda_{\gamma} \otimes a \mid \gamma \in \Gamma, a \in \mathcal{M}^{\alpha}(\gamma)\right\}$, 
the automorphism $\beta_{\gamma}$ for $\gamma \in \Gamma$ is given by $\operatorname{Ad}\left(\lambda_{\hat{\imath}(\gamma)^{-1}} \otimes \lambda_{\gamma^{-1}} \otimes 1\right)$ and the automorphism $\tau_{\mu}$ for $\mu \in \hat{E}$ is given by $\operatorname{Ad}\left(\lambda_{\mu} \otimes 1 \otimes 1\right)$. Hence

$$
\begin{gathered}
\mathcal{R}=\left(\left\{M_{\langle t, \cdot\rangle} \otimes M_{\langle g, \cdot\rangle} \otimes 1 \mid t \in E, g \in G\right\} \cup\left\{1 \otimes \lambda_{\gamma} \otimes a \mid \gamma \in \Gamma, a \in \mathcal{M}^{\alpha}(\gamma)\right\}\right)^{\prime \prime} \\
\cap\left\{\lambda_{\hat{\iota}(\gamma)^{-1}} \otimes \lambda_{\gamma^{-1}} \otimes 1 \mid \gamma \in \Gamma\right\}^{\prime} .
\end{gathered}
$$

Let $U$ be the unitary on $L^{2}(\hat{E} \times \Gamma)$ given by $(U \xi)(\mu, \gamma)=\xi\left(\hat{\imath}(\gamma) \mu^{-1}, \gamma\right)$ for $\mu \in \hat{E}$, $\gamma \in \Gamma$, so $U^{*}=U$. Then

$$
\begin{aligned}
U\left(M_{\langle t, \cdot\rangle} \otimes M_{\langle g, \cdot\rangle}\right) U & =M_{\overline{\langle t, \cdot\rangle}} \otimes M_{\langle\hat{\iota}(t) g, \cdot\rangle}, \\
U\left(1 \otimes \lambda_{\gamma}\right) U & =\lambda_{\hat{\iota}(\gamma)} \otimes \lambda_{\gamma} \\
U\left(\lambda_{\hat{\iota}(\gamma)^{-1}} \otimes \lambda_{\gamma^{-1}}\right) U & =1 \otimes \lambda_{\gamma^{-1}} \\
U\left(\lambda_{\mu} \otimes 1\right) U & =\lambda_{\mu^{-1}} \otimes 1
\end{aligned}
$$

and

$$
\begin{aligned}
U \mathcal{R} U= & \left(\left\{M_{F} \otimes M_{f} \otimes 1 \mid F \in L^{\infty}(\hat{E}), f \in \ell^{\infty}(\Gamma)\right\}\right. \\
& \left.\cup\left\{\lambda_{\hat{\iota}(\gamma)} \otimes \lambda_{\gamma} \otimes a \mid \gamma \in \Gamma, a \in \mathcal{M}^{\alpha}(\gamma)\right\}\right)^{\prime \prime} \cap\left\{1 \otimes \lambda_{\gamma^{-1}} \otimes 1 \mid \gamma \in \Gamma\right\}^{\prime} \\
= & \left(\left\{M_{F} \otimes 1 \otimes 1 \mid F \in L^{\infty}(\hat{E})\right\} \cup\left\{\lambda_{\hat{\iota}(\gamma)} \otimes \lambda_{\gamma} \otimes a \mid \gamma \in \Gamma, a \in \mathcal{M}^{\alpha}(\gamma)\right\}\right)^{\prime \prime} .
\end{aligned}
$$

Moreover, the automorphism $\tau_{\mu}$ of $\mathcal{R}$ corresponds to the automorphism $\operatorname{Ad}\left(\lambda_{\mu^{-1}} \otimes 1 \otimes 1\right)$ of $U \mathcal{R} U$. This is precisely the picture for $\widetilde{\mathcal{P}}$ and $(\alpha \circ \iota)_{\mu}^{\hat{\mu}}$ obtained above.

Corollary 3.4. $\quad$ Let $\mathcal{M}$ be a purely infinite von Neumann algebra having separable predual and $\varphi$ a n.f.s. almost periodic weight on $\mathcal{M}$. Let $\Gamma$ be the subgroup of $\mathbf{R}_{+}^{*}$ generated by the point spectrum of $\Delta_{\varphi}$ and let $(\mathcal{N}, \Gamma, \beta)$ giving rise to $\mathcal{M} \simeq \mathcal{N} \rtimes_{\beta} \Gamma$ be the discrete decomposition of $\mathcal{M}$ associated to $\varphi$. Let $\left(\widetilde{\mathcal{N}}, \mathbf{R}_{+}^{*}, \widetilde{\beta}\right)$ giving rise to $\mathcal{M} \simeq \widetilde{\mathcal{N}} \rtimes_{\widetilde{\beta}} \mathbf{R}_{+}^{*}$ be Takesaki's continuous decomposition. Then the action $\widetilde{\beta}$ of $\mathbf{R}_{+}^{*}$ on $\widetilde{\mathcal{N}}$ is the action induced up to $\mathbf{R}_{+}^{*}$ from the action $\beta$ of $\Gamma$ on $\mathcal{N}$.

Proof. Apply Proposition 3.3 with $\alpha$ and $\iota$ as in Section 1.3. 


\section{A model for the one-parameter trace-scaling action on the injective $\mathrm{II}_{\infty}$ factor.}

Lemma 4.1. Let $\mathcal{N}_{i}$ be a von Neumann algebra with almost periodic n.f.s. weight $\psi_{i},(i=1,2)$. Let $\mathcal{M}=\mathcal{N}_{1} \otimes \mathcal{N}_{2}$ be the tensor product von Neumann algebra and $\varphi=\psi_{1} \otimes \psi_{2}$ the tensor product weight. Then $\varphi$ is an almost periodic n.f.s. weight on $\mathcal{M}$ and the point spectrum of $\Delta_{\varphi}$ is the product of the point spectra of $\Delta_{\psi_{1}}$ and $\Delta_{\psi_{2}}$. Let $\Gamma_{i}$ (respectively $\Gamma$ ), taken with discrete topology, be the subgroup of $\mathbf{R}_{+}^{*}$ generated by the point spectrum of $\Delta_{\psi_{i}}$ (respectively $\left.\Delta_{\varphi}\right)$, so $\Gamma=\Gamma_{1} \Gamma_{2}$, and let $G_{i}=\widehat{\Gamma}_{i}$ (respectively $\left.G=\widehat{\Gamma}\right)$. Let $\alpha_{i}($ respectively $\alpha)$ be the action of $G_{i}$ on $\mathcal{N}_{i}$ (respectively $G$ on $\left.\mathcal{M}\right)$ as in Section 1.3. Then for $\gamma \in \Gamma$, the spectral subspace is

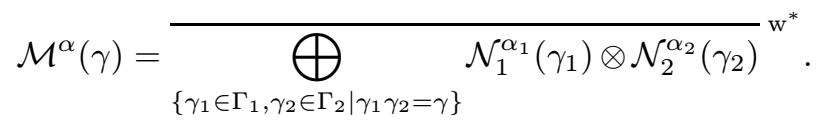

Proof. From $L^{2}(\mathcal{M}, \varphi)=L^{2}\left(\mathcal{N}_{1}, \psi_{1}\right) \otimes L^{2}\left(\mathcal{N}_{2}, \psi_{2}\right)$ and $\Delta_{\varphi}=\Delta_{\psi_{1}} \otimes \Delta_{\psi_{2}}$ one sees that $\varphi$ is almost periodic and has the desired point spectrum. Moreover, from the characterization in Lemma 1.3.3, one sees that $\supseteq$ holds in (9). But by Lemma 1.2.3, the direct sum of $\mathcal{N}_{1}^{\alpha_{1}}\left(\gamma_{1}\right) \otimes \mathcal{N}_{2}\left(\gamma_{2}\right)$ over all $\gamma_{1} \in \Gamma_{1}$ and $\gamma_{2} \in \Gamma_{2}$ is dense in $\mathcal{M}$, so equality must hold in (9).

Theorem 4.2. Let $0<\mu_{1}, \mu_{2}<1$ be such that $\log \left(\mu_{1}\right) / \log \left(\mu_{2}\right)$ is irrational. Let $\Gamma$ be the multiplicative subgroup of $\mathbf{R}_{+}^{*}$ generated by $\mu_{1}$ and $\mu_{2}$, let $\mathcal{N}$ be the injective $I I_{\infty}$ factor and let $\beta$ be an action of $\Gamma$ on $\mathcal{N}$ such that $\operatorname{Tr}_{\mathcal{N}} \circ \beta_{\gamma}=\gamma \operatorname{Tr}_{\mathcal{N}} \forall \gamma \in \Gamma$, where $\operatorname{Tr}_{\mathcal{N}}$ is the n.f.s. trace on $\mathcal{N}$. Then the action of $\mathbf{R}_{+}^{*}$ induced up from the action $\beta$ of $\Gamma$ on $\mathcal{N}$ is the one-parameter trace-scaling action of $\mathbf{R}_{+}^{*}$ on the injective $I I_{\infty}$ factor. (See Definition 3.1.)

Remark 4.3. Since a trace-scaling action $\beta$ of $\Gamma$ on $\mathcal{N}$ is relatively easy to construct, Theorem 4.2 gives an accessible picture of the one-parameter tracescaling action on the injective $\mathrm{II}_{\infty}$ factor, heuristically as translation on the fixed point subalgebra of $L^{\infty}\left(\mathbf{R}_{+}^{*}, \mathcal{N}\right)$ under a pair of commuting automorphisms (see Definition 3.1)

Proof of Theorem 4.2. By [12], all trace-scaling actions $\beta$ of $\Gamma$ on $\mathcal{N}$ are outer conjugate, so it suffices to show the theorem for a particular one. Let $\lambda_{i}=\left(1+\mu_{i}\right)^{-1}$ and let $\mathcal{M}^{\lambda_{i}}$ be Powers' factor with Powers' state $\omega_{i}=\omega_{\lambda_{i}}[\mathbf{1 3}]$. It is well known ( $c f .[\mathbf{1 4}]$, Section 5) that the centralizer $\mathcal{M}_{\omega_{i}}^{\lambda_{i}}$ is the hyperfinite $\mathrm{II}_{1}$-factor, $R$, and the point spectrum of $\Delta_{\omega_{i}}$ equals $\left\{\mu_{i}^{n} \mid n \in \mathbf{Z}\right\}$. Consider the AFD (approximately finite dimensional), purely infinite factor $\mathcal{M}=\mathcal{M}^{\lambda_{1}} \otimes \mathcal{M}^{\lambda_{2}}$ and let $\varphi=\omega_{1} \otimes \omega_{2}$ be the tensor product state. Then by Lemma $4.1 \varphi$ is 
almost periodic, the point spectrum of $\Delta_{\varphi}$ is $\Gamma=\left\{\mu_{1}^{n_{1}} \mu_{2}^{n_{2}} \mid n_{1}, n_{2} \in \mathbf{Z}\right\}$ and the centralizer $\mathcal{M}_{\varphi}=R \otimes R \simeq R$ is a factor. Now by Proposition 2.12, the discrete decomposition of $\mathcal{M}$ associated to $\varphi$ is a trace-scaling action of $\Gamma$ on the injective $\mathrm{II}_{\infty}$ factor. By the factoriality of the centralizer and Connes [4], 3.2.7., it follows that $\mathcal{M}$ is a type $\mathrm{III}_{1}$ factor, so Takesaki's continuous decomposition is the one-parameter trace-scaling action of $\mathbf{R}_{+}^{*}$ on the injective type $\mathrm{II}_{\infty}$ factor. Now Corollary 3.4 finishes the proof.

Remark 4.4. A more concrete picture of the one-parameter trace-scaling action in terms of operators on Hilbert space can be had from the proof of Theorem 4.2. In [14], Section 5, Takesaki showed that $\omega_{i}$ is a homogeneous, periodic state. Thus, by general results of the same paper, there is an isometry, $v_{i}$, in the spectral subspace $\mathcal{M}_{\omega_{i}}^{\lambda_{i}}\left(\mu_{i}^{-1}\right)$ such that

$$
\mathcal{M}_{\omega_{i}}^{\lambda_{i}}\left(\mu_{i}^{n}\right)= \begin{cases}M_{\omega_{i}}^{\lambda_{i}} v_{i}^{-n} & \text { if } n \leq 0 \\ \left(v_{i}^{*}\right)^{n} M_{\omega_{i}}^{\lambda_{i}} & \text { if } n \geq 0 .\end{cases}
$$

Keeping in mind that the centralizers $\mathcal{M}_{\omega_{i}}^{\lambda_{i}}$ are copies of the hyperfinite $\mathrm{II}_{1}$ factor, $R$, and noting that the spectral subspaces of the tensor product are

$$
\mathcal{M}_{\varphi}\left(\mu_{1}^{n_{1}} \mu_{2}^{n_{2}}\right)=\mathcal{M}_{\omega_{1}}^{\lambda_{1}}\left(\mu_{1}^{n_{1}}\right) \otimes \mathcal{M}_{\omega_{2}}^{\lambda_{2}}\left(\mu_{2}^{n_{2}}\right),
$$

one can use (10) to describe the spectral subspaces of $\mathcal{M}$ under $\varphi$. Let $\mathcal{H}$ be the Hilbert space on which $\mathcal{M}$ acts. Then by Proposition 3.2, the injective $\mathrm{II}_{\infty}$ factor is the von Neumann algebra on $L^{2}\left(\mathbf{R}_{+}^{*}\right) \otimes \mathcal{H}$ generated by

$$
\left(L^{\infty}\left(\mathbf{R}_{+}^{*}\right) \otimes 1\right) \cup\left\{\lambda_{\gamma} \otimes a \mid \gamma \in \Gamma, a \in \mathcal{M}_{\varphi}(\gamma)\right\}
$$

and the one-parameter trace-scaling action is given by $\operatorname{Ad}\left(\lambda_{t} \otimes 1\right)$ for $t \in \mathbf{R}_{+}^{*}$, i.e., translation by $t$ on the first component.

5. Another model for the one-parameter trace-scaling action on the injective $\mathbf{I I}_{\infty}$ factor. We now explain a model which was described to the author by V. F. R. Jones, and depends on work of P.-L. Aubert. The usual (linear) action of $S L(2, \mathbf{Z})$ on $\mathbf{R}^{2}$ preserves Lebesque measure. Let $\mathcal{N}$ be the crossed product von Neumann algebra of $L^{\infty}\left(\mathbf{R}^{2}\right)$ by the action of $S L(2, \mathbf{Z})$. From Lebesque measure on $\mathbf{R}^{2}$, one gets a n.f.s. trace on $\mathcal{N}$. In [2], Aubert showed the somewhat surprising fact that $\mathcal{N}$ is the injective $\mathrm{II}_{\infty}$ factor. Then for $t \in \mathbf{R}_{+}^{*}$, the dilation of $\mathbf{R}^{2}$ by $t$ gives an automorphism, $\alpha_{t}$, of $\mathcal{N}$ that scales the trace by $t$. So $t \mapsto \alpha_{t}$ is the one-parameter trace-scaling action of $\mathbf{R}_{+}^{*}$ on the injective $\mathrm{II}_{\infty}$ factor. 


\section{REFERENCES}

[1] W. ARVESon, On groups of automorphisms of operator algebras, J. Funct. Anal. 15 (1974), 217-243.

[2] P.-L. Aubert, Deux actions de $S L(2, \mathbf{Z})$, Théorie Ergodic, L'Enseignement Mathématique 29, pp. 39-46.

[3] L. BARnett, Free product von Neumann algebras of type III, Proc. Amer. Math. Soc. 123 (1995), 543-553.

[4] A. Connes, Une classification des facteurs de type III, Ann. scient. Éc. Norm. Sup. $4^{\mathrm{e}}$ serie 6 (1973), 133-252.

[5] Classification of injective factors, Ann. of Math. 104 (1976), 73-115.

[6] - Almost periodic states and factors of type $I I_{1}$, J. Funct. Anal. 16 (1974), 415-445.

[7] K. Dykema, Factoriality and Connes' invariant $T(\mathcal{M})$ for free products of von Neumann algebras, J. reine angew. Math. 450 (1994), 159-180.

[8] _ Free products of finite dimensional and other von Neumann algebras with respect to non-tracial states, (Preprint).

[9] J. Feldman, C. C. Moore, Ergodic equivalence relations, cohomology, and von Neumann algebras. I, Trans. Amer. Math. Soc. 234 (1977), 289-324.

[10] U. HAAGERUP, Connes' bicentralizer problem and uniqueness of the injective factor of type $I I I_{1}$, Acta. Math. 158 (1987), 95-148.

[11] R. V. Kadison, J. R. Ringrose, Fundamentals of the Theory of Operator Algebras, Academic Press, 1986.

[12] A. Ocneanu, Actions of Discrete Amenable Groups on von Neumann Algebras, Lecture Notes in Mathematics 1138, Springer-Verlag, 1985.

[13] R. T. POWERS, Representations of uniformly hyperfinite algebras and their associated von Neumann rings, Ann. Math. 86 (1967), 138-171.

[14] M. TAKESAKI, The structure of a von Neumann algebra with a homogeneous periodic state, Acta. Math. 131 (1973), 79-121.

[15] Duality for crossed products and the structure of von Neumann algebras of type III, Acta. Math. 131 (1973), 249-310.

This work was supported by a National Science Foundation Postdoctoral Fellowship.

Department of Mathematics

University of California

Berkeley, California 94720-3840

E-MAIL: dykema@fields-ws020.UWaterloo.ca

Received: June 13th, 1994; revised: June 19, 1995. 